\title{
MREEP: Multihop Residual Energy based Energy Efficient Routing Protocol for HWSN
}

\author{
Muhammad Rizwan ${ }^{1,}$, Muhammad S. Nisar ${ }^{2, b}$ and Hongbo Jiang ${ }^{1, c}$ \\ ${ }^{1}$ School of Electronic Information and Communications, HUST, Wuhan 430074, China; \\ ${ }^{2}$ Wuhan National Laboratory for Optoelectronics, HUST, Wuhan 430074, China. \\ a1201422058@hust.edu.cn, ${ }^{b}$ I201322185@hust.edu.cn, chongbojiang@hust.edu.cn
}

Keywords: Heterogeneous Wireless Sensor Network (HWSN), Cluster Head (CH), Stability Period.

\begin{abstract}
Energy conversation of sensor nodes for maximization of network life is the most important design goal while developing an energy efficient routing protocol for wireless sensor networks. Heterogeneity takes the advantage of different types of sensor nodes and improves the network life and energy efficiency. In the energy conversation solutions, most of the researches have been done on energy efficient solutions regardless of the multihop short distance communication between cluster heads (CHs) and sink. To solve this problem, a novel Multihop Residual Energy based Energy Efficient Routing Protocol (MREEP) for Heterogeneous WSN has been proposed in order to enhance the stability period and longevity of the network. In MREEP, CH computes the optimal multihop path and transmits the data towards the sink via its nearest CHs. MREEP uses residual energy based election of CHs with novel double layered election probability mechanism. The simulation results show that our proposed routing scheme can achieve longer sensor network life, better energy efficiency as compare to conventional routing protocols.
\end{abstract}

\section{Introduction}

Over recent years, the Wireless Sensor Networks (WSNs) have drawn more attention worldwide. The advancement of smart sensors technology has been facilitated by Micro-Electro-Mechanical Systems (MEMS) technology. A WSN is comprised of distributed autonomous sensor nodes. The sensor nodes have the capability to sense, gather and measure the information. The sensed data is transmitted to sink via possible multihop communication. WSNs are used in many industrial application areas, including healthcare application and home automation [1]. HWSNs networks have become more popular because of the advantages of using HWSN with different resources in order to meet the demands of various applications which have been studied in the recent literature [2].

The important challenges in the organization of wireless sensor network are energy efficiency, stability and improvement in network life time because the sensor nodes are limited in energy, storage capacity and computational power. The battery of nodes cannot be changed or recharged due to dense deployment in the harsh environment. Because sensor nodes have limited energy, so it is important to introduce the energy conservation techniques in order to extend the life time of WSNs [3].

Clustering is an efficient technique to address the challenges related to energy consumption and scalability. Cluster based routing techniques are very helpful in solving the sensor nodes energy constraints by reducing the cost of data aggregation and transmission of sensed data before transmitting to sink [4].

\section{Heterogeneous Network Model}

In this Model, $\mathrm{n}$ sensor nodes are randomly and uniformly deployed in MxM square meter sensing region. Base station is located at the center of sensing area. There are two types of sensor nodes with different initial energy used in this HWSN model. These two types of sensor nodes are normal and advance nodes. Let $E_{0}$ is the initial energy of the normal nodes and $E_{0} .(1+\alpha)$ be the initial energy of $\mathrm{m}$ fraction of the advance nodes, where $\alpha$ means that advance nodes contain $\alpha$ times more initial 
energy than normal nodes (1-m)n [6]. Total Initial energy of HWSN model is given by: $\quad E_{\text {total }}=N(1+\alpha m) E_{0}$

In our work, we use the radio energy dissipation model similar to [5]. Free space and multipath fading channel are used in this energy model. The amount of energy required to transmit L bits message over a distance $d$ from one node to another node is given by:

$$
E_{T x}(L, d)=\left\{\begin{array}{l}
L \times \text { Elec }+L \times E_{f s} \times d^{2} \text { if } d<d_{0} \\
L \times \text { Elec }+L \times E_{m p} \times d^{4} \text { if } d<d_{0}
\end{array}\right.
$$

Elec is the dissipated energy to run the transmitter or receiver. The parameters $E_{f s}$ and $E_{m p}$ is the amount of energy dissipation which depends upon the distance $\mathrm{d}_{0}$ which is given as $d_{0}=\sqrt{E_{f s} / E_{m p}}$. In SEP [6] the energy dissipation for cluster head to sink is fixed regardless of the distance while in our purposed routing scheme energy is dissipated according to the distance from cluster head to sink. In order to verify our results we have modified the energy dissipation in SEP in order to incorporate consideration of distance from cluster head to sink. To receive the $L$ bits message, dissipated energy is equal to $E_{r x}(L)=L . E_{r x-e l e c}$.

\section{MREEP: Multihop Residual Energy based Routing Protocol}

This MREEP is a clustering algorithm for two level heterogeneous wireless sensor networks. In MREEP, $\mathrm{n}$ sensor nodes are randomly distributed in the $\mathrm{M} \times \mathrm{M}$ sensing region. The distance from node to $\mathrm{CH}$ or sink is $\leq \mathrm{d}_{0}$. The energy dissipated by $\mathrm{CH}$ and non- $\mathrm{CH}$ in a single round is defined as:

$$
\begin{aligned}
& E_{C H}=L . E l e c\left(\frac{n}{k}-1\right)+L . E_{D A} \frac{n}{k}+L . E l e c+L . E_{f S} d_{t o B S}^{2} \\
& E_{\text {nonCH }}=L .\left(\text { Elec }+E_{f S} \times d_{\text {toCH }}^{2}\right)
\end{aligned}
$$

Where $E_{D A}$ is defined as the energy required for data aggregation in a round, $\mathrm{k}$ is defined as number of clusters, $d_{t o c H}$ is defined as the average distance between nodes and $\mathrm{CH}$ and $d_{t o B s}$ is defined as average distance between cluster head and base station. Where. The total expended energy can be calculated as follows:

$$
E_{\text {total }}=L .\left(2 n E_{\text {elec }}+n E_{D A}+k E_{f S} d_{\text {toBS }}^{2} \times n E_{f S} d_{\text {toCH }}^{2}\right)
$$

The optimal number of clusters can be calculated by $k_{\text {opt }}=\sqrt{E_{f s} / E_{m p}} \times \sqrt{n / 2 \pi} \times \sqrt{M / d_{\text {toBs }}^{2}}$ the average distance $d_{t o B S}$ can be calculated as follows [6]: $d_{t o B S}=0.765 \times M / 2$ and the optimal probability of node to become a cluster head in a round is defined as: $p_{\text {opt }}=k_{\text {opt }} / n$. Let $p_{n r m l}, p_{a d v}$ represent the weighted election probability for normal and advance nodes. The average number of $\mathrm{CHs}$ per round per epoch is equal to $n$. $(1+\alpha m) p_{n r m l}$. The weighted probability of normal and advance nodes can be calculated as:

$$
p_{n r m l}=\frac{p_{o p t}}{1+\alpha . m}, p_{a d v}=\frac{p_{o p t}}{1+\alpha . m} \times(1+\alpha)
$$

In the two-level HWSN, we replace the reference $p_{\text {opt }}$ value with the weighted probabilities given in Eq. 6. Therefore, $p_{i}$ is changed into:

$$
p_{i}=\left\{\begin{array}{c}
\frac{p_{\text {opt }} E_{i}(r)}{(1+\alpha . m) \bar{E}(r)} \text { if } S_{i} \text { is normal node } \\
\frac{p_{\text {opt }} E_{i}(r)(1+\alpha)}{(1+\alpha . m) \bar{E}(r)} \text { if } S_{i} \text { is advanced node }
\end{array}\right.
$$

The T $\left(S_{i}\right)$ probability threshold by which node $S_{i}$ uses to determine whether it can be elected as $\mathrm{CH}$ in a round is defined as:

$$
\mathrm{T}\left(S_{i}\right)=\left\{\begin{array}{cl}
\frac{p_{i}}{1-p_{i}\left(r \bmod 1 / p_{i}\right)} & \text { if } S_{i} \in G^{\prime} \\
0 & \text { otherwise }
\end{array}\right.
$$

Where $G^{\prime}$ is defined as the set of either normal or advance nodes that are not elected as CHs within the last $1 / p_{n r m l}$ or $1 / p_{a d v}$ rounds.

Proposed MREEP: In this research work, a novel Multihop Residual Energy based Energy Efficient Routing Protocol (MREEP) for HWSN has been proposed in order to enhance the stability period and network lifetime of the sensor network. In MREEP, the sensor nodes which are nearer to 
sink transmit the data directly to sink for saving energy. CH computes the optimal multihop path and transmits the data towards the sink via its nearest cluster heads. In MREEP, if the residual energy of advance nodes is less than normal nodes, both will have equal probability to become $\mathrm{CH}$. The residual energy based election of CHs with double layered election probability mechanism. This novel multilayer election probability mechanism plays a vital role to enhance the network lifetime of the sensor network.

\section{Simulation and Results Analysis}

In this section, we evaluate the performance of purposed routing scheme. We use $100 \times 100$ square meter sensing region of 100 sensor nodes. Sink is located in the middle of sensing field. The following performance parameters are used to evaluate the performance of purposed routing scheme.

Stability Period. Stability Period is the time interval from when the network operation starts until death of first node.

Instability Period. Instability Period is the time interval starting from the death of $1^{\text {st }}$ node till death of last node.

Network Lifetime. Network lifetime is the time interval between the network operation starts until the death of last node.

Number of Alive Nodes. This will measure the number of live nodes that are still active and part of network.

Number of Dead Nodes. This will measure the number of dead nodes that are inactive and utilized all the energy.

We compare it with LEACH and SEP in the same heterogeneous setting. The packet size is 500 bytes and initial energy is $\mathrm{E}_{0}=0.5 \mathrm{~J}$. The other key parameters used in simulation are given in Table 1 .

Table 1 Simulation Parameters

\begin{tabular}{cl}
\hline Parameter & Value \\
\hline Area & $100 \mathrm{~m} \mathrm{x} 100 \mathrm{~m}$ \\
$N$ & 100 \\
$E_{\text {elec }}$ & $50 \mathrm{~nJ} / \mathrm{bit}$ \\
$E_{D A}$ & $5 \mathrm{~nJ} / \mathrm{bit} / \mathrm{message}$ \\
$E_{m p}$ & $10 \mathrm{pJ} / \mathrm{bit} / \mathrm{m}^{4}$ \\
$E_{f s}$ & $0.0013 \mathrm{pJ} / \mathrm{bit} / \mathrm{m}^{2}$ \\
$P_{\text {opt }}$ & 0.1 \\
$d_{o}$ & $87.7 \mathrm{~m}$ \\
\hline
\end{tabular}

We divide our experiments in two cases. In the first case: $\alpha=2, \mathrm{~m}=0.2$ and rounds $=8000$, for second case: $\alpha=4, \mathrm{~m}=0.1$ and rounds $=15000$. In the first case, we can observe that MREEP has increased the stability period of the network as compared to LEACH and SEP. In the LEACH and SEP, the death of first node occurs at $609^{\text {th }}$ round and $1056^{\text {th }}$ round respectively while in MREEP first node died at $1081^{\text {st }}$ round. Similarly, in the second case, we can see that in LEACH and SEP death of first node occurred at $545^{\text {th }}$ round and $986^{\text {th }}$ round while in MREEP the death of first node occurred at

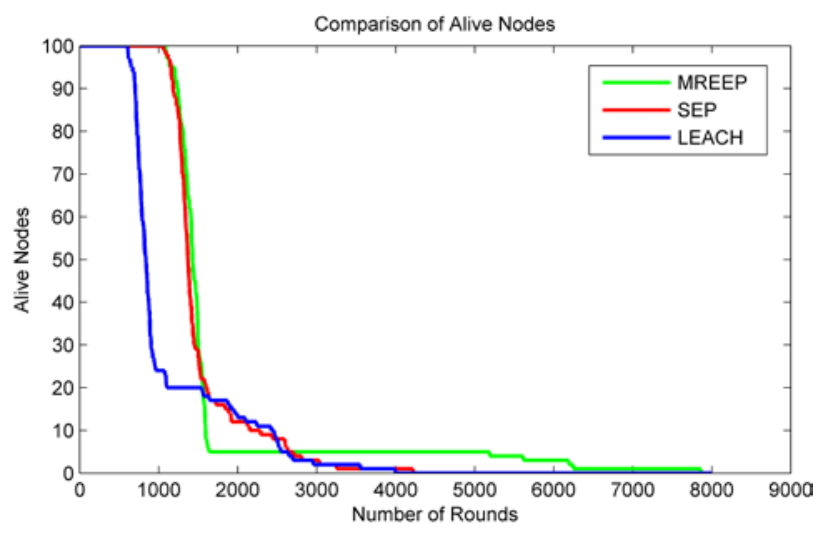

Fig. 1. $(\alpha=2, m=0.2)$, Rounds $=8000$

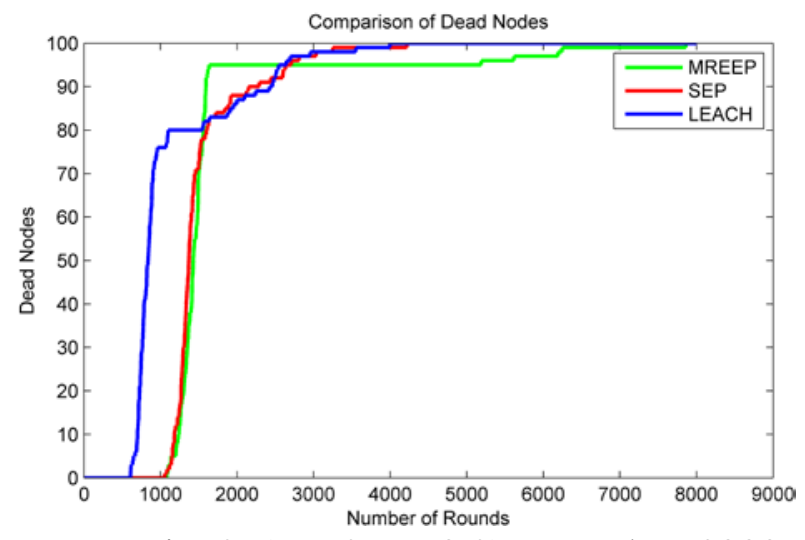

Fig. 2. $(\alpha=2, m=0.2)$, Rounds $=8000$ 
$1202^{\text {nd }}$ round. In fig. 1 and fig. 2, we can observe that MREEP has increased the stability period of the network as compared to LEACH and SEP. We can verify that the stability period of network is improved in the case of MREEP as compare to LEACH and SEP. In first case, we can observe that MREEP has significantly increased the instability period of the network as compared to LEACH and SEP. In LEACH and SEP all nodes died at $3996^{\text {th }}$ round and $4218^{\text {th }}$ round respectively while in MREEP all nodes died after 7861 rounds. Similarly in second case, we can see that in LEACH and SEP all nodes die at $7161^{\text {st }}$ round and $6117^{\text {th }}$ round respectively while in MREEP all nodes died after13374 rounds. The results show that MREEP improves total network lifetime by $86.7 \%$ compared to LEACH and $118 \%$ as compared to SEP. It is thus clear that network lifetime has improved in the case of MREEP as compare to LEACH and SEP as shown in fig. 2 and fig.4.

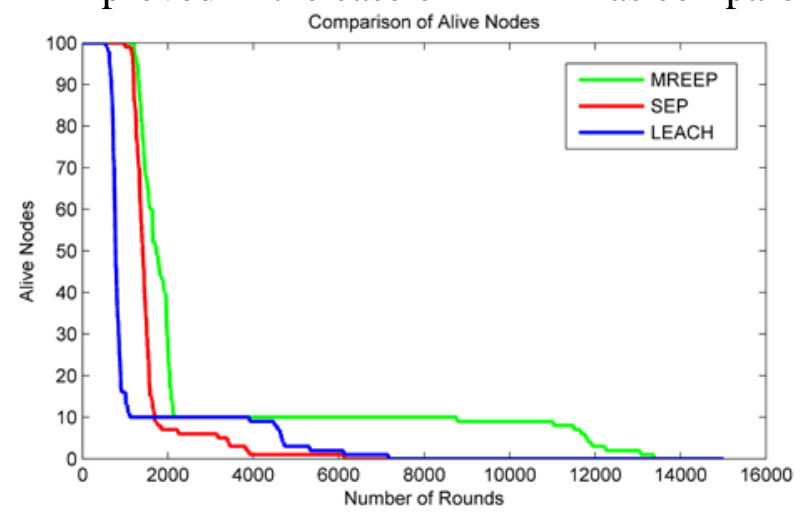

Fig. 3. $(\alpha=4, m=0.1)$, Rounds $=15000$

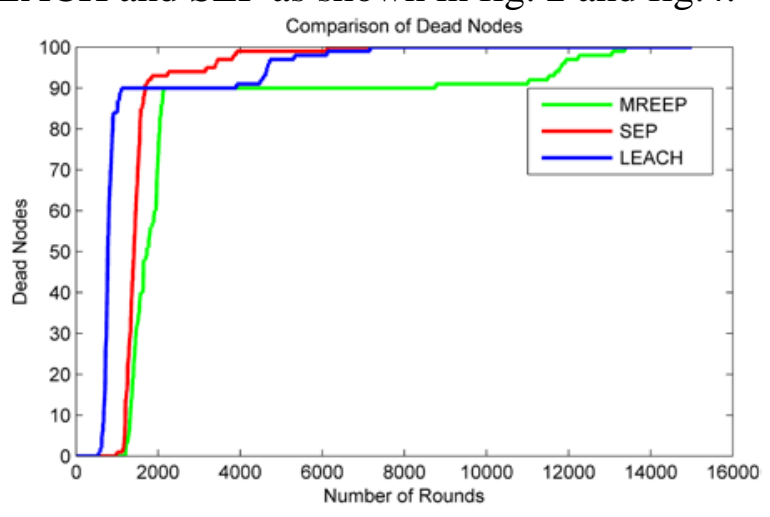

Fig. 4. $(\alpha=4, m=0.1)$, Rounds $=15000$

\section{Conclusion}

The simulation results proved that the purposed routing protocol, MREEP, significantly increased the stability period and network life time of the sensor network as compared to SEP and LEACH. The results indicate that MREEP can economize the energy consumption of the entire sensor network and extend the longevity of the network. In future, MREEP can be extended to incorporate more types of nodes enhancing heterogeneity of the network and level of hierarchy in the clustered HWSN.

\section{References}

[1] I.F. Akyildiz, W.Su, Y. Sankarasubmmaiam, E.Cayirci, “A Survey on Sensor Networks,” IEEE Comm. Magazine, pp.102-114, Aug 2002.

[2] R Kumar, V. Tsiatsis, M.B. Srivastava, Computation Hierarchy for In-Network Processing, Proceedings of 2nd ACM International Workshop on Wireless Networks and Applications, San Diego, CA, 68-77, 2003.

[3] Kemal Akkaya and Mohamed Younis, "A Survey on Routing Protocols for Wireless Sensor Networks,” Ad Hoc Networks, Vol. 3, No. 3, pp. 325-349, May 2005.

[4] J. Al-Karaki, and A. Kamal, "Routing Techniques in Wireless Sensor Networks: A Survey,” IEEE Communications Magazine, Vol. 11, no. 6, Dec. 2004, pp. 6 -28.

[5] W. Heinzelman, A. Chandrakasan, and H. Balakrishnan, "EnergyEfficient Communication Protocols for Wireless Microsensor Networks,” Proc. Of 33th Hawaiian International Conference on Systems Science, 2000.

[6] Smaragdakis, I Matta, A Bestavros. SEP: A Stable Election Protocol for clustered heterogeneous wireless sensor networks. in: Second International Workshop on Sensor and Actor Network Protocols and Applications (SANPA). 2004. 